\title{
Mineralogy of four Itokawa particles collected from the first touchdown site
}

\author{
Takaaki Noguchi ${ }^{1 *}$, John C Bridges ${ }^{2}$, Leon J Hicks², Steven J Gurman², Makoto Kimura ${ }^{3}$, Takahito Hashimoto ${ }^{4}$, \\ Mitsuru Konno ${ }^{4}$, John P Bradley ${ }^{5}$, Ryuji Okazaki ${ }^{6}$, Masayuki Uesugi ${ }^{7}$, Toru Yada ${ }^{7}$, Yuzuru Karouji ${ }^{7}$, Masanao Abe ${ }^{7}$, \\ Tatsuaki Okada ${ }^{7}$, Takuya Mitsunari ${ }^{3}$, Tomoki Nakamura ${ }^{8}$ and Hiroyuki Kagi ${ }^{9}$
}

\begin{abstract}
Four Itokawa particles collected from the first touchdown site were mineralogically investigated by optical microscopy, micro-Raman ( $\mu$-Raman) spectrometry, scanning electron microscopy (SEM), electron microprobe analysis (EPMA), X-ray absorption spectroscopy (XAS), and transmission electron microscopy (TEM). Their mineralogy has an affinity to that of LL6 chondrites based on micro-Raman spectroscopy, EPMA, and XAS analyses. However, the space weathering rims on them are less developed than those observed on the Itokawa particles collected from the second touchdown site. Solar flare tracks are rarely observed in the four particles, whose number densities were lower than those observed in the Itokawa particles from the second touchdown site.
\end{abstract}

Keywords: Itokawa; XANES; EPMA; TEM; Space weathering; Solar flare tracks

\section{Background}

In 2005, the Hayabusa spacecraft sampled twice from the Muses-C Regio of the asteroid (25143) Itokawa filled with myriad of pebbles. The distance between two touchdown sites is about $100 \mathrm{~m}$ (Nakamura et al. 2014). The Itokawa particles investigated during the initial analyses in 2011 were collected from the second touchdown site (sample number: RB-QD04-xxxx), and those from the first touchdown site (sample number: RA-QD02$\operatorname{xxxx})$ were investigated in the first announcement of opportunity (AO) consortium, which includes this study. The Itokawa particles from the second touchdown site showed mineralogical and isotopic affinities to LL5-6 chondrites (Nakamura et al. 2011; Yurimoto et al. 2011; Nakamura et al. 2012; Tsuchiyama et al. 2011, 2014; Nakashima et al. 2013). Nakamura et al. (2014) reported that olivine, pyroxene, and plagioclase in six particles from the first touchdown site have chemical compositions similar to those from the second touchdown site. As a part of the first AO consortium, four Itokawa particles from the first touchdown site were allocated to us.

\footnotetext{
* Correspondence: tnoguchi@artsci.kyushu-u.ac.jp

${ }^{1}$ Faculty of Arts and Science, Kyushu University, 744 Motooka, Nishi-ku, Fukuoka 819-0395, Japan

Full list of author information is available at the end of the article
}

Although the sampling sites are located just about $100 \mathrm{~m}$ away from each other within the MUSES-C Regio, the reflectance spectra at the MUSES-C Regio show a variation in the relative reflectance associated with wavelength (Ishiguro et al. 2007). Because it is thought that the degree of spectral reddening is related to the abundance of nanophase $\mathrm{Fe}^{0}$ on the surface material of Itokawa (e.g., Binzel et al. 2001; Hiroi et al. 2006), the microstructures of the external surface of the Itokawa particles from the first touchdown site are worth comparing with those from the second touchdown site.

Because the number of Itokawa particles collected from the first touchdown site is limited, six particles allocated to Nakamura et al. (2014), four particles to Tsuchiyama et al. (2014), and another four particles to this study, mineralogical data of the four Itokawa particles would contribute to clarify the mineralogy of the Itokawa particles from the first touchdown site.

The four Itokawa particles collected from the first touchdown site were mineralogically investigated by optical microscopy, micro-Raman ( $\mu$-Raman) spectrometry, scanning electron microscopy (SEM), electron microprobe analysis (EPMA), X-ray absorption spectroscopy (XAS), and transmission electron microscopy (TEM). Among these methods, XAS is the newly applied technique for the analysis of the Itokawa samples. 
If the affinity of Itokawa particles to LL5-6 chondrites is common, the redox state experienced by the four Itokawa particles in their parent body should be almost identical to that in LL5-6 chondrites. Therefore, the relative abundance of $\mathrm{Fe}^{3+}$ and $\mathrm{Fe}^{2+}$ ions in ferromagnesian silicates, which reflect the redox states, in the particles from Itokawa were measured and compared with those in ferromagnesian silicates in an LL chondrite. We used Tuxtuac LL5 chondrite for comparison. The X-ray absorption near-edge structure (XANES) of Fe K-edge is an X-ray absorption spectroscopy (XAS) technique used to compare Itokawa to chondrite meteorite samples.

\section{Methods}

Four Itokawa particles collected during the first touchdown were allocated to our group (Table 1). The particles were stored in a sealed box in the clean chamber no. 2, in which the sample curation of the Itokawa particles is going on in an ultrapure $\mathrm{N}_{2}$ atmosphere, and were transferred to and opened in an $\mathrm{N}_{2}$-purged glove box. Both the chamber and the glove box were installed in the Planetary Material Sample Curation Facility of JAXA (PMSCF/JAXA). All the samples were embedded in epoxy resin in the glove box to avoid contact with earth's atmosphere. Dew-point temperature was around $-40^{\circ} \mathrm{C}$ during embedding and curing. After cure of epoxy, they were stored in an $\mathrm{N}_{2}$-gas-filled aluminum-laminated plastic bag and transferred to Ibaraki University for further processing and analyses.

The cross sections at the external surface of the four particles were observed by using Hitachi HF-3300 cold field-emission (FE) TEM (Hitachi High-Technologies Co., Tokyo, Japan) equipped with EDAX energy dispersive X-ray spectrometer (EDS) (Philips, Amsterdam, Netherlands) at Hitachi High-Technologies Co. An additional TEM observation was performed by using FEI Titan TEM (FEI, Hillsboro, OR, USA) at Lawrence Livermore National Laboratory, USA, and JEOL JEM-2100 TEM (JEOL Ltd., Tokyo, Japan) at Ibaraki University. Solar flare tracks in the Itokawa samples were obtained by a method

Table 1 List of the Itokawa particles investigated in this study and their constituent minerals

\begin{tabular}{ll}
\hline Sample & Minerals \\
\hline RB-QD04-0008 & Olivine, high-Ca pyroxene, troilite \\
RB-QD04-0011 & Olivine \\
RB-QD04-0015 & Olivine \\
RB-QD04-0024 & Low-Ca pyroxene, plagioclase, troilite \\
\hline
\end{tabular}

The samples in the bag were ultramicrotomed into 90-nm-thick sections by Reichert Ultracut-N (Reichert-Jung, Vienna, Austria) at Ibaraki University for TEM. Each of the samples was taken out from the bag, just prior to ultramicrotomy, and exposure to the air was shorter than $1 \mathrm{~h}$. Details of the ultramicrotomy were presented in Noguchi et al. (2014). similar to the weak-beam dark-field method (e.g., Williams and Carter 2009).

Raman spectra of potted butts of the four samples (the epoxy-embedded sample remained after ultramicrotomy) were measured with a $\mu$-Raman spectrometer (JASCO Inc., NRS-3100, Tokyo, Japan) at Ibaraki University to identify minerals. The wavelength of excitation laser is $532 \mathrm{~nm}$. The beam diameter of the laser is $2 \mu \mathrm{m}$. The peak position of the strongest Raman shift peak of metallic Si was calibrated to $520 \mathrm{~cm}^{-1}$. Measuring time was $40 \mathrm{~s} \times 2$, and the laser power was from 1 to $10 \mathrm{~mW}$. After micro-Raman measurements, the potted butts were coated by carbon, and their textures were studied with a focused ion beam combined with scanning electron microscope (FIB-SEM) JEOL JIB-4501 equipped with JED-2100 EDS (JEOL Ltd., Tokyo, Japan) at Ibaraki University. Mineral chemistry was measured with a JEOL JXA-8530F FE-EPMA (JEOL Ltd., Tokyo, Japan) at JEOL Co. Acceleration voltage and probe current were $15 \mathrm{kV}$ and $9 \mathrm{nA}$, respectively. ZAF-oxide correction was applied to calculate chemical compositions of minerals.

The potted butts of the four Itokawa particles and a polished thin section of the Tuxtuac LL5 chondrite were analyzed using the Beamline I18 X-ray micro-focus spectroscopy beamline (Diamond Light Source Ltd, Oxfordshire, UK) at the Diamond Light Source, Oxfordshire, UK. Diamond is a 3-GeV synchrotron with ring currents of approximately $250 \mathrm{~mA}$. Energy selection with fractional energy resolutions of $10^{-4}$ to $10^{-5}$ are achieved with an Si (111) and (311) double crystal monochromator, respectively. An Si drift vortex detector was used to measure the X-ray fluorescence and absorbance of elements with $Z>40$. Fe K-edge X-ray absorption spectroscopy (XAS) was performed on approximately 2.5$\mu \mathrm{m}$ spots and also with a mapping routine over approximately $5 \times 40 \mu \mathrm{m}$ areas. Typical experimental conditions used for XAS were 1-s integration at each $5.0 \mathrm{eV}$ energy step up to approximately 7,100 eV, followed by a higher resolution of $0.1 \mathrm{eV}$ energy steps over the XANES features up to $7,150 \mathrm{eV}$, and continuing over the EXAFS region with steps of 2 to $4 \mathrm{eV}$ up to 7,660 eV. The errors of the Fe K-edge positions were $\pm 0.05 \mathrm{eV}$.

After the XAS, FIB sections of RB-QD04-0008 and RB-QD04-0024 were prepared from their potted butts by JEOL JIB-4501 FIB-SEM (JEOL Ltd., Tokyo, Japan) and an ultra-low energy Ar ion milling machine Fischione NanoMill (E.A. Fischione Instruments, Inc., Corporate Circle Export, PA, USA) at Ibaraki University. Because the external surfaces of the Itokawa particles existed at the bottom of these potted butt samples, the ultrathin sections and the FIB sections were prepared from the opposite sides of these particles although the thickness of these particles were unknown. The FIB sections were observed by FE-TEM JEOL JEM-2800 at JEOL Co. and JEOL 
JEM-2100 TEM (JEOL Ltd., Tokyo, Japan) at Ibaraki University.

\section{Results}

Description of the four Itokawa particles

The optical photomicrographs of the four allocated particles before ultramicrotomy and backscattered electron (BSE) images of their potted butts after ultramicrotomy are presented in Figures 1 and 2, respectively. RB-QD040008 is an approximately $40-\mu \mathrm{m}$-wide composite grain. An approximately $20-\mu \mathrm{m}$ equigranular olivine attaches a high-Ca pyroxene similar in form and size (Figure 1a). Both of the olivine and high-Ca pyroxene in this sample contain small opaque grains $(<2 \mu \mathrm{m}$ in diameter: black dots in Figure 1a). Three of them were cropped out to the surface of the potted butt and are enriched in Fe and S. Therefore, the opaque minerals include troilite (Figure 2a). Both RB-QD04-0011 and RB-QD04-0015 are 35 and $46 \mu \mathrm{m}$ wide, respectively, and composed only of anhedral olivine including small amounts of opaque minerals $(<2 \mu \mathrm{m}$ in diameter) (Figure $1 \mathrm{~b}, \mathrm{c})$. No opaque mineral was exposed to the surfaces of their potted butts (Figure 2b,c). RB-QD04-0024 is a flat and thin $(<10 \mu \mathrm{m})$ approximately $50-\mu \mathrm{m}$-long grain composed of low-Ca pyroxene and plagioclase containing with abundant small $(<2 \mu \mathrm{m}$ in diameter) opaque minerals. The opaque minerals appeared on the potted butts are troilite (Figure 2d). Olivine, low-Ca pyroxene, and high-Ca pyroxene in these Itokawa particles do not show clear undulatory extinction. Because low-Ca pyroxene in RB-QD04-0024 does not display polysynthetic twinning, it is expected to be orthopyroxene, which is supported by $\mu$-Raman spectrometry, which will be described in the next section. The optical features of plagioclase in RB-QD04-0024 were not able to be determined due to the small grain size.

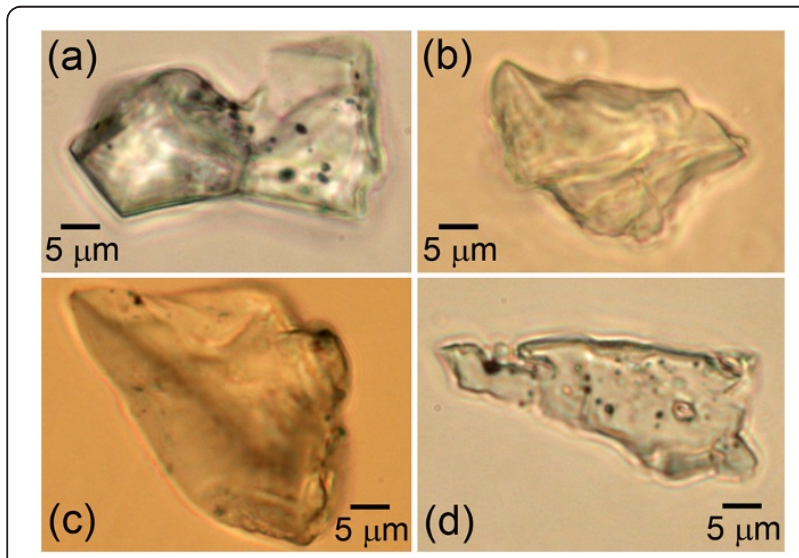

Figure 1 Optical images of the four Itokawa particles investigated in this study. All of them were stored in Room B of the sample catcher. (a) RB-QD04-0008, (b) RB-QD04-0011, (c) RB-QD04-0015, and (d) RB-QD04-0024

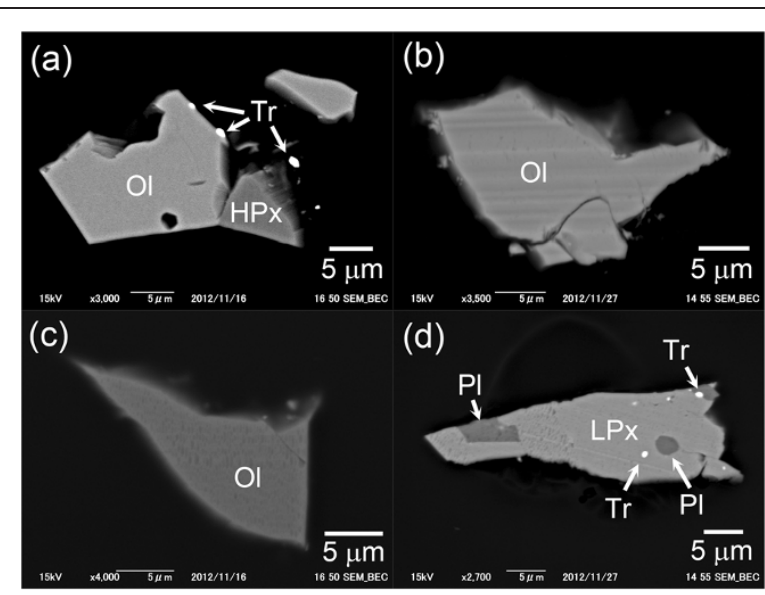

Figure 2 Backscattered electron images of the potted butts of the four Itokawa particles investigated in this study. All of them were stored in Room B of the sample catcher. (a) RB-QD04-0008, (b) RB-QD04-0011, (c) RB-QD04-0015, and (d) RB-QD04-0024. Ol, olivine; LPx, low-Ca pyroxene; Pl, plagioclase.

\section{Raman spectroscopy}

Raman shift peaks assignable to olivine, low-Ca pyroxene, and high-Ca pyroxene were obtained (Table 2). However, no peaks of plagioclase in RB-QD04-0024 were detected, and we did not identify whether it is maskelynite or not. Olivine grains in RB-QD04-0008, RBQD04-0011, and RB-QD04-0015 show a doublet peak. According to Kuebler et al. (2006), the shorter and the longer wavenumber peaks of the olivine doublet are called DB1 (doublet peak \#1) and DB2 (doublet peak \#2), respectively. The peak positions of DB1 and DB2 are displayed in Table 2 and plotted in a DB2 position vs DB1 position diagram (Figure 3). A hatched area in Figure 3, which appears as a narrow curved band along the $\mathrm{Fo}_{70}$ curve, shows the range of forsterite ( $\mathrm{Fo}$ ) $\mathrm{mol} \%$ obtained by EPMA for these olivine grains. Forsterite mol\% estimated by the DB1 and DB2 positions is approximately 10 percentage points higher than the actual Fo mol\%. Low-Ca pyroxene in RB-QD04-0024 shows a doublet near approximately $670 \mathrm{~cm}^{-1}\left(677.6\right.$ and $\left.656.1 \mathrm{~cm}^{-1}\right)$, which is indicative of orthopyroxene (Wang et al. 2001).

\section{Chemical compositions of olivine and pyroxenes}

Chemical compositions of olivine, low-Ca pyroxene, and high-Ca pyroxene were obtained by EPMA. Representative data are shown in Table 3. A histogram of Fa mol\% (100-Fo mol\%) is shown in Figure 4. Olivine in the four

Table 2 Raman shift peak positions DB1 and DB2 of olivine

\begin{tabular}{lll}
\hline Sample & DB1 position $\left(\mathbf{c m}^{-1}\right)$ & DB2 position $\left(\mathbf{c m}^{-\mathbf{1}}\right)$ \\
\hline RB-QD04-0008 & 820.8 & 850.8 \\
RB-QD04-0011 & 820.7 & 851.5 \\
RB-QD04-0015 & 821.1 & 851.3 \\
\hline
\end{tabular}




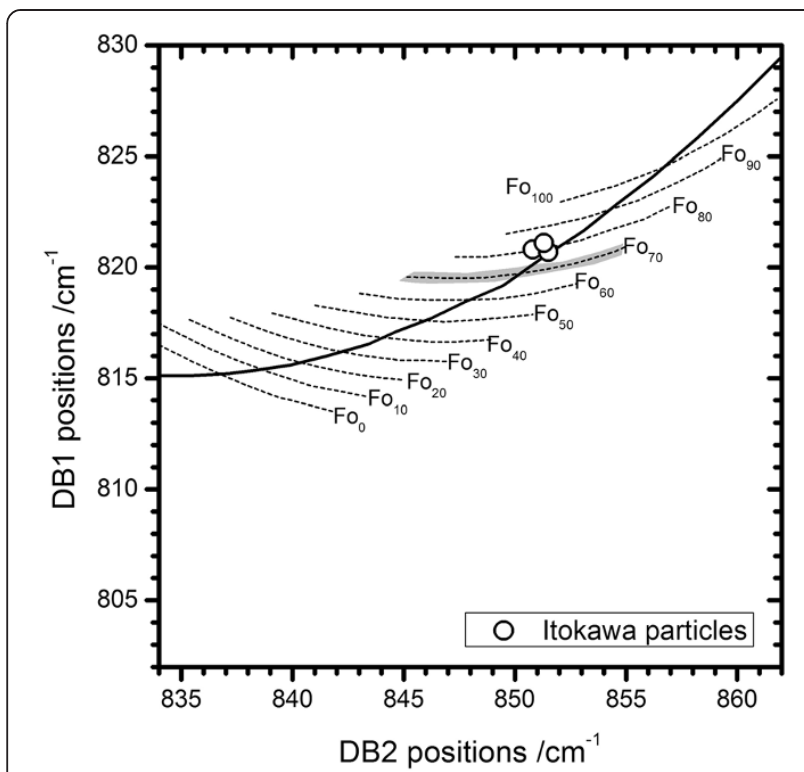

Figure 3 Relationships between the positions of the olivine doublet peaks, DB1 and DB2 in the three Itokawa particles. A hatched area shows the range of forsterite mol\% in olivine in the three samples that were obtained by an electron microprobe analyzer. The dotted curves show contours of different Fo mol\%, and a black solid curve is the best fit curve between the positions of DB1 and DB2 by Kuebler et al. (2006).

Itokawa grains are homogeneous, and their $\mathrm{Fa}$ mol\% are within the range of those in the equilibrated grains investigated during the initial analysis $\left(\mathrm{Fa}_{27.1-30.7}\right.$ : Nakamura et al. 2011). Average and standard deviation of Fa mol\% of olivine in RB-QD04-0008, RB-QD04-0011, and RB-QD040015 are $30.5 \pm 0.6(n=3), 29.7 \pm 0.3(n=3)$, and $31.0 \pm 0.7$ $(n=2)$, respectively.
Chemical compositions of low-Ca and high-Ca pyroxenes are plotted on a pyroxene quadrilateral diagram (Figure 5). Average and standard deviation of En and Wo mol\% of low-Ca pyroxene in RB-QD04-0024 are $74.3 \pm 0.3$ and $2.2 \pm 0.02(n=3)$, respectively. En and Wo mol\% of high-Ca pyroxene in RB-QD04-0008 are 46.1 and 41.9 , respectively. Chemical composition of plagioclase was not obtained due to its small size.

\section{XANES analysis}

\section{Fe K-edge XANES of olivine}

The Fe K-edge XANES plots of olivine in three Itokawa particles show a clear absorption pre-edge feature, which is common to olivine in the Tuxtuac LL5 $\left(\mathrm{Fo}_{71.0 \pm 0.3}, n=25\right)$ and terrestrial San Carlos olivine (approximately $\mathrm{Fo}_{90}$ ) (Figure 6a). The edge positions and the pre-edge centroid positions of olivine in the three Itokawa particles are $7,119.5$ to $7,119.8 \mathrm{eV}$ and 7,112.5 to 7,112.6 eV, respectively. These values are indistinguishable from those in terrestrial olivine and olivine in Tuxtuac (Table 4).

\section{Fe K-edge XANES of pyroxene}

The edge position and the pre-edge centroid position of low-Ca pyroxene in the Itokawa grain 0024 are 7,119.7 to 7,119.8 and 7,112.6 eV, respectively (Figure 6b). These values are indistinguishable from those measured in low-Ca pyroxene in the Tuxtuac LL5 chondrite $\left(\mathrm{Wo}_{1.5 \pm 0.3} \mathrm{En}_{75.1 \pm 0.5}\right.$, $n=15$ ): 7,119.8 and 7,112.6 eV, respectively (Table 4).

\section{TEM observation}

\section{Surface modification}

Surface modification at the external surfaces of the four Itokawa particles was investigated using FE-TEM. High-

Table 3 Representative chemical compositions of olivine and pyroxenes in the four Itokawa particles

\begin{tabular}{|c|c|c|c|c|c|}
\hline & \multicolumn{5}{|c|}{ Sample } \\
\hline & RB-QD04-0008 & RB-QD04-0008 & RB-QD04-0011 & RB-QD04-0015 & RB-QD04-0024 \\
\hline & \multicolumn{5}{|c|}{ Mineral } \\
\hline & Olivine & High-Ca pyroxene & Olivine & Olivine & Low-Ca pyroxene \\
\hline $\mathrm{SiO}_{2}$ & 37.64 & 52.00 & 36.91 & 37.33 & 54.87 \\
\hline $\mathrm{TiO}_{2}$ & b. d. & 0.42 & b. d. & b. d. & 0.05 \\
\hline $\mathrm{Al}_{2} \mathrm{O}_{3}$ & b. d. & 0.45 & b. d. & b. d. & 0.08 \\
\hline $\mathrm{Cr}_{2} \mathrm{O}_{3}$ & b. d. & 0.77 & b. d. & b. d. & 0.15 \\
\hline $\mathrm{FeO}$ & 27.07 & 7.49 & 26.18 & 27.14 & 15.19 \\
\hline $\mathrm{MnO}$ & 0.50 & 0.21 & 0.43 & 0.42 & 0.40 \\
\hline $\mathrm{MgO}$ & 34.12 & 16.13 & 35.30 & 34.65 & 27.23 \\
\hline $\mathrm{CaO}$ & b. d. & 20.41 & b. d. & b. d. & 1.11 \\
\hline $\mathrm{Na}_{2} \mathrm{O}$ & b. d. & 0.43 & b. d. & b. d. & b. d. \\
\hline $\mathrm{K}_{2} \mathrm{O}$ & b. d. & b. d. & b. d. & b. d. & b. d. \\
\hline Total wt.\% & 99.33 & 98.31 & 98.82 & 99.54 & 99.08 \\
\hline Mol\% end members & $\mathrm{Fo}_{69.2}$ & $\mathrm{Wo}_{41.9} \mathrm{En}_{46.1}$ & $\mathrm{FO}_{70.6}$ & $\mathrm{Fo}_{69.5}$ & $\mathrm{Wo}_{2.2} \mathrm{En}_{74.5}$ \\
\hline
\end{tabular}

Fo, forsterite; Wo, wollastonite; En, enstatite; b. d., below detection limit. 


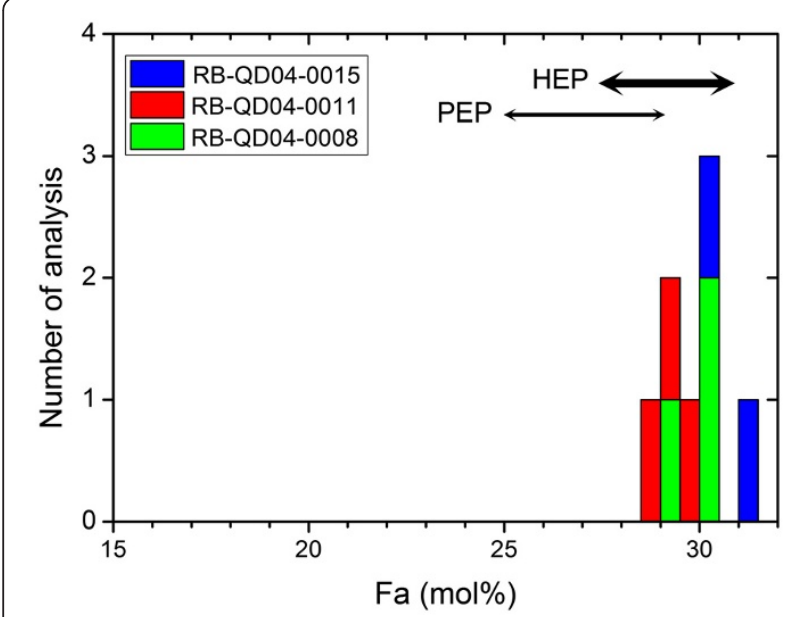

Figure $4 \mathrm{~A}$ histogram of forsterite $\mathrm{mol} \%$ in olivine included in three Itokawa particles. The ranges of those in highly equilibrated particles (HEP) and in poorly equilibrated particles (PEP) investigated by Nakamura et al. (2011) are also indicated by arrows for comparison.

resolution bright-field TEM images show that two Itokawa particles RB-QD04-0015 and RB-QD04-0024 have an amorphous zone (labeled 'I' in Figure 7c,d) and a partially amorphized zone (labeled 'II' in Figure 7c,d), while those of the other two particles (RB-QD04-0008 and RBQD04-0011) display sharp lattice fringes to the surface (Figure 7a,b). The thickness of the amorphous layer is 5 to $8 \mathrm{~nm}$ (indicated by double-headed arrows marked 'I' in Figure $7 \mathrm{c}, \mathrm{d})$. In both cases, approximately 2-nm-size poorly crystalline nanophase inclusions (indicated by

(a)

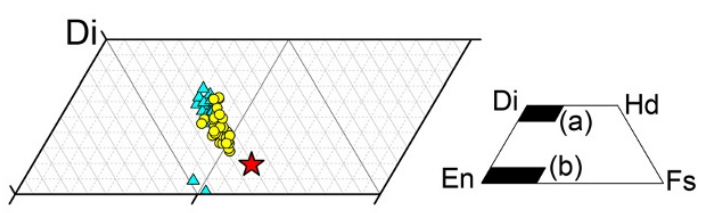

(b)

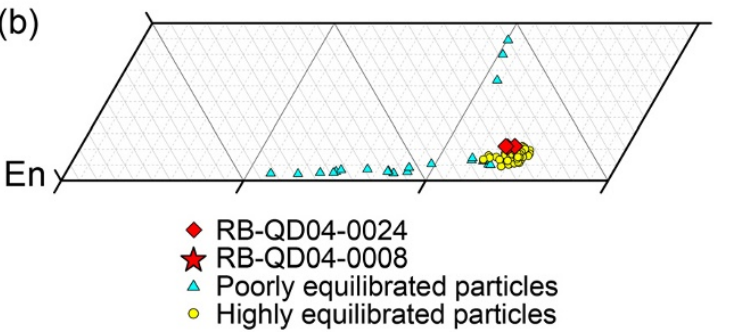

Figure 5 Chemical compositions of low- and high-Ca pyroxenes in RB-QD04-0008 and RB-QD04-0024 plotted on a pyroxene quadrilateral diagram. (a) An enlarged diagram showing a chemical composition of high-Ca pyroxene in RB-QD04-0008. (b) An enlarged diagram showing those of low-Ca pyroxene in RB-QD04-0024. Chemical compositions of pyroxenes included in highly equilibrated and poorly equilibrated particles investigated by Nakamura et al. (2011) are also plotted for comparison. arrows) exist on the external surface. By comparing Figure 7, high-angle annular dark field-scanning transmission electron microscope (HAADF-STEM) images and elemental distribution maps (Figure 8), the nanophases form a densely arranged array at the external surface, which is indicated by arrows in HAADF-STEM images of Figure 8. $\mathrm{Mg}$ and Fe elemental maps indicate that the inclusions are enriched in these elements (Figure 8). Figure 8 shows that the nanophase inclusions in RB-QD04-015 are enriched in S as well as Mg and Fe.

Just below the nanophase-rich layer, there are $\mathrm{Si}$ enrichment and depletion in $\mathrm{Mg}$ and $\mathrm{Fe}$ in both of RBQD04-0015 and RB-QD04-0024. In the case of RBQD04-0024, the lower part of the amorphous layer (labeled as B) is more electron transparent than the upper part (labeled as A), suggestive of lower density. In addition, a slight enrichment of $\mathrm{Al}$ is detected in $\mathrm{RB}-$ QD04-0015.

Below the amorphous layer, underlying olivine and low-Ca pyroxene show partial amorphization indicated by arrows marked 'II' in Figure 7c,d. The partially amorphized olivine and low-Ca pyroxene contains 2-to-4-nmsize nanophases (Figure 7e,f). Their lattice fringes range from 0.19 to $0.20 \mathrm{~nm}$, suggestive of (110) of $\alpha$-iron metal (Noguchi et al. 2014). The amorphous layer containing nanophase $(\mathrm{Fe}, \mathrm{Mg}) \mathrm{S}$ and the underlying partially amorphized minerals containing nanophase $\mathrm{Fe}$ are similar to the composite rims observed on the Itokawa particles investigated in the initial analysis (Noguchi et al. 2014). The composite rim is composed of a redeposition zone and a partially amorphized zone, called zones I and II, respectively (Noguchi et al. 2014).

\section{Surface modification of two different areas within a single particle}

To assess whether surface modification was heterogeneously distributed on a single Itokawa particle, two FIB sections were prepared from two Itokawa particles. By using both ultrathin sections and FIB sections, surface modification on the opposite sides of these particles was able to be examined. In both cases, the same mineral existed on the opposite sides. One is RB-QD04-0008, which has almost no surface modification, and the other is RB-QD04-0024 having a composite rim. Figure 9 shows dark-field images of the FIB sections of these particles. The FIB section of the former does not show any surface modification, and the latter has surface modification of approximately $50 \mathrm{~nm}$ thick (indicated by an arrow), which corresponds to the total thickness of the composite rim.

\section{Solar flare tracks}

The solar flare tracks in the four Itokawa particles were searched based on the dark-field TEM observation. The 

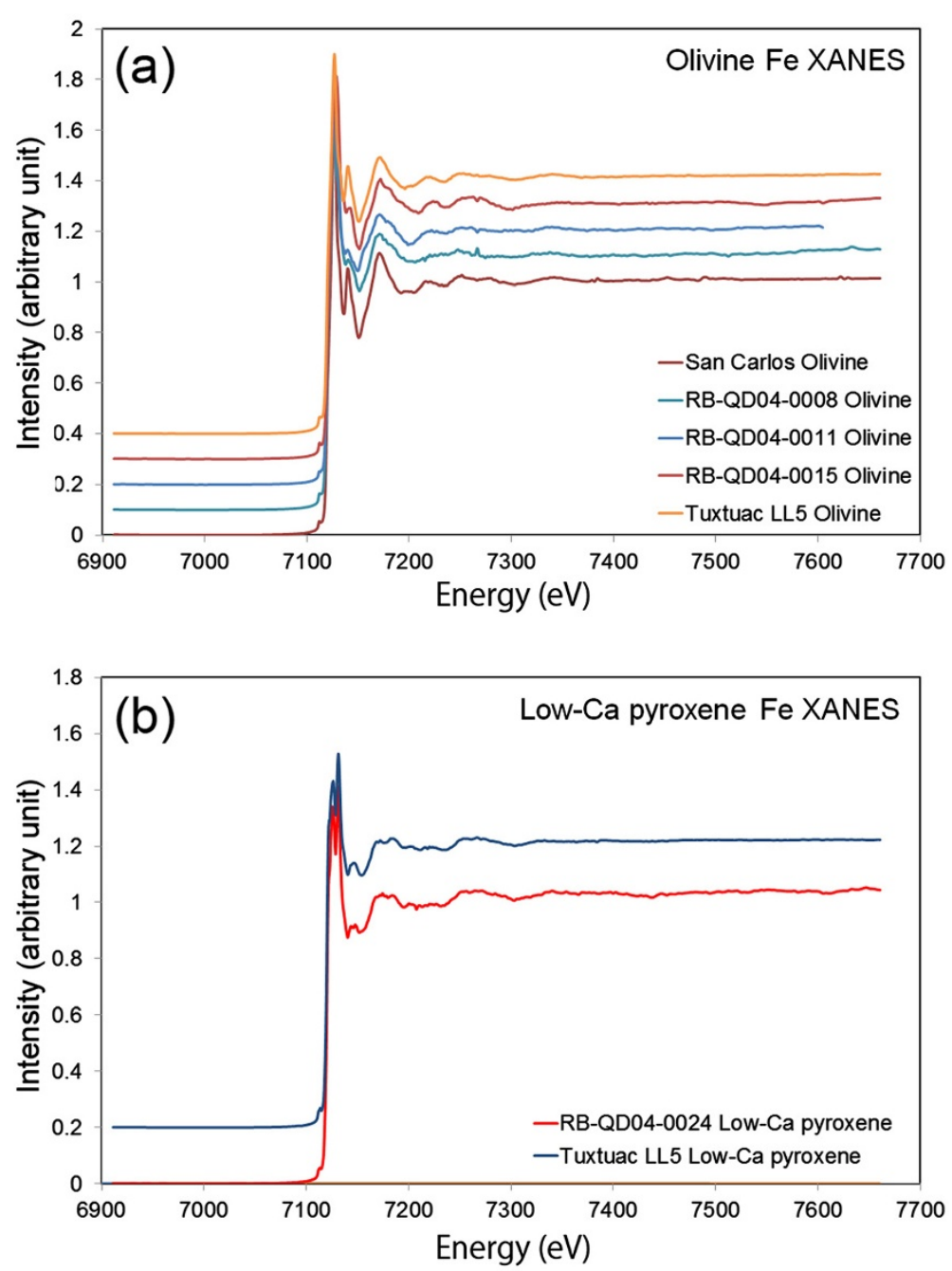

Figure 6 Fe K-edge XANES spectra. (a) Fe K-edge of olivine in the three Itokawa particles: RB-QD04-0008, RB-QD04-0011, and RB-QD04-0015, Those of olivine in the Tuxtuac LL5 chondrite and San Carlos olivine are also plotted for comparison. (b) Fe K-edge of low-Ca pyroxene in RB-QD04-0024 and the Tuxtuac LL5 chondrite.

Table 4 Fe XANES edge positions and pre-edge centroid positions in olivine and pyroxenes in the four Itokawa particles and Tuxtuac LL5 chondrite

\begin{tabular}{llcc}
\hline Sample & Mineral & Edge position $(\mathbf{e V})$ & $\begin{array}{c}\text { Pre-edge } \\
\text { centroid }(\mathbf{e V})\end{array}$ \\
\hline RB-QD04-0008 & Olivine & $7,119.5$ & $7,112.6$ \\
RB-QD04-0011 & Olivine & $7,119.5$ & $7,112.6$ \\
RB-QD04-0015 & Olivine & $7,119.8$ & $7,112.5$ \\
Tuxtuac LL5 & Olivine & $7,119.5$ & $7,112.7$ \\
RB-QD04-0008 & High-Ca pyroxene & $7,119.4$ & $7,112.6$ \\
RB-QD04-0024 & Low-Ca pyroxene & $7,119.7$ & $7,112.6$ \\
Tuxtuac LL5 & Low-Ca pyroxene & $7,119.8$ & $7,112.6$ \\
\hline
\end{tabular}

tracks are rare in the Itokawa particles investigated in this study. Figure 10a shows a possible solar flare track in olivine in an ultrathin section of RB-QD04-0015. The number density of the solar flare tracks was estimated to be $<1 \times 10^{9}$ tracks $/ \mathrm{cm}^{2}$. A composite rim of $<50 \mathrm{~nm}$ thick is seen on the external surface. On the other hand, the number density of the solar flare tracks is obviously higher in some Itokawa particles collected at the first touchdown site that were investigated in the initial analysis. For example, Figure 10b shows a dark-field image of low-Ca pyroxene in a FIB section of RA-QD02-0009, in which solar flare tracks are indicated by arrows. The track number density was estimated to be $2 \times 10^{9} \mathrm{~cm}^{-2}$. This particle has a vesicular composite rim of approximately $80 \mathrm{~nm}$ (Noguchi et al. 2014). 


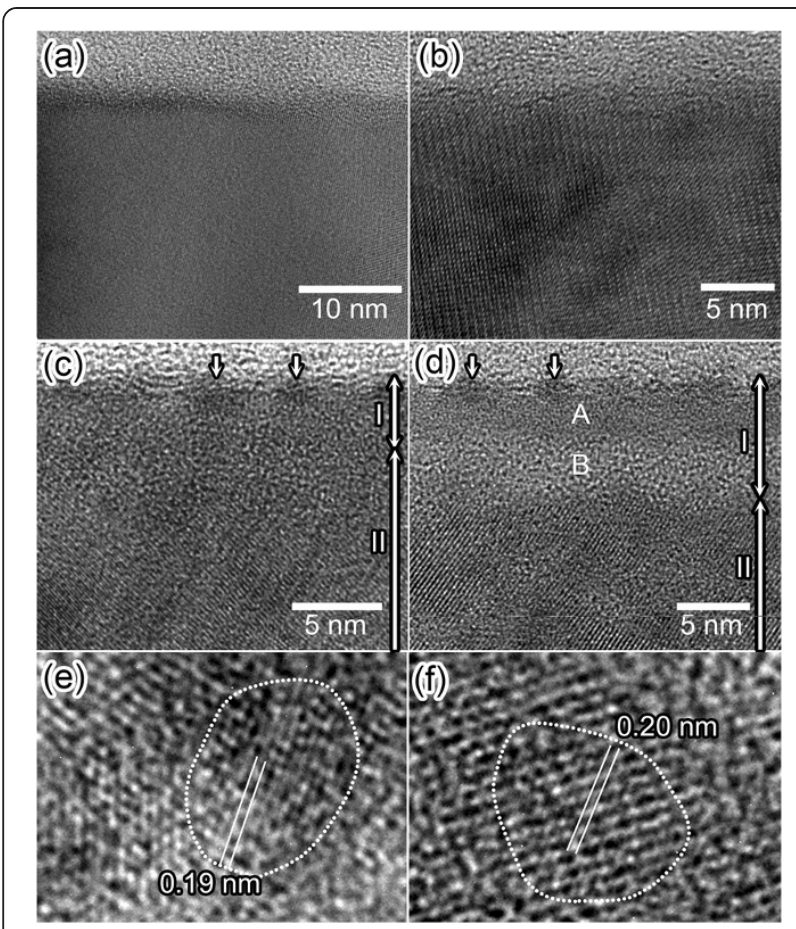

Figure 7 Bright-field TEM images of ultrathin sections of four Itokawa particles. (a) Olivine in RB-QD04-0008, (b) olivine in RB-QD04-0011, (c) olivine in RB-QD04-0015, and (d) low-Ca pyroxene in RB-QD04-0024. In (c) and (d), the amorphous surface zone is indicated by double-headed arrows marked 'I.' Nanophases on the external surface, which appear as dark speckles, were indicated by arrows. In (c), the lower part of the amorphous layer (B) is more electron transparent than the upper part (A). Single-headed arrows marked 'II' indicate the partially amorphized zone. Nanophase Fe inclusions showing (e) 0.19 - and (f) 0.20 -nm lattice fringes in the partially amorphized zone of olivine and low-Ca pyroxene, respectively. They are indicated by dotted closed curves.

\section{Discussion}

\section{Morphology and mineralogy of RB-QD4-0008}

Because their sizes (approximately 35 to approximately $50 \mu \mathrm{m})$ are comparable to the smallest grains investigated during the initial analysis (whole size range: approximately 30 to approximately $180 \mu \mathrm{m}$ ) (Nakamura et al. 2011, 2014), the petrography of the Itokawa grains investigated in this study is simpler than that of those investigated in the initial analysis. Among them, RBQD04-0008 has a unique morphology although the other Itokawa particles are fragments composed of an olivine grain (RB-QD04-0011 and RB-QD04-0015) or low-Ca pyroxene and plagioclase (RB-QD04-0024). The particle is composed of partially-connected equigranular olivine and high-Ca pyroxene. Itokawa particles having a morphology similar to these particles have been reported previously (Nakamura et al. 2011; Matsumoto et al. 2012; Noguchi et al. 2014). For example, RA-QD02-0060 is composed of a large twinned low-Ca clinopyroxene and small olivine and vesiculated plagioclase. In this particle, the dislocation density of olivine grains is variable. This fact suggests that such porous aggregate particles were formed by the recrystallization of mineral particles, each of which has a different history.

The Enstatite (En) and Wo contents (mol\%) in the highCa pyroxene in RB-QD04-0008 are 46.1 and 41.9, respectively, which are outside the range of the En and Wo contents in high-Ca pyroxene in the highly equilibrated grains investigated in the initial analysis (Nakamura et al. 2011) (Figure 5). The mol\% of Wo is similar to those of high-Ca pyroxene in LL6 chondrites (McSween and Patchen 1989). By considering the above discussion, RBQD04-0008 may have been formed by recrystallization and it contains mineral fragments that experienced thermal metamorphism as high as most LL6 chondrites experienced.

\section{Mineralogy of low-Ca pyroxene in RB-QD4-0024}

As described in the 'Raman spectroscopy' section, the low-Ca pyroxene in RB-QD04-0024 is orthopyroxene. The average Wo mol\% of the low-Ca pyroxene is 2.2, which is higher than that of the equilibrated Itokawa

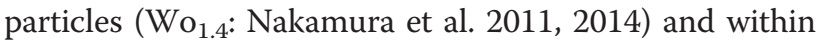
the range of LL6 chondrites ( $\mathrm{Wo}_{1.7-2.6}$ : McSween and Patchen 1989). The highly equilibrated particles (HEP) from the second touchdown site were regarded as being comparable with LL5-6 chondrites based on the Wo mol\% of both low- and high-Ca pyroxene (Nakamura et al. 2011, 2014), and the low-Ca pyroxene in RB-QD04-0024 is plotted at the highest Wo mol\% of low-Ca pyroxene in the HEP (Figure 5). These data suggest that RB-QD04-0024 is a mineral fragment that experienced thermal metamorphism comparable with the typical LL6 chondrites. Although we investigated only four Itokawa particles in this study, each particle experienced a different metamorphic history, which is consistent with the previous studies of Itokawa particles (Nakamura et al. 2011, 2014).

\section{Space weathering and solar flare track density}

Two of the four Itokawa particles investigated in this study show textural modification at the external surfaces based on TEM observation. They have amorphous surface layers and underlying partially amorphized olivine or low-Ca pyroxene (Figures 7 and 8). In RB-QD040015, the amorphous surface layer contains $\mathrm{Al}$, which is not contained in olivine. These features correspond to those of the composite rims, which have probably been formed by space weathering (Noguchi et al. 2011, 2014).

The rims of RB-QD04-0015 and RB-QD04-0024 do not have blisters that are lenticular bubbles formed by ion implantation (e.g., Igarashi et al. 2002). On the other hand, blisters were observed in 3 among 12 Itokawa particles in the initial analysis (Noguchi et al. 2014). Although the number of particles investigated in this study is limited, the degrees of space weathering may have 
Figure 8 HAADF-STEM images and elemental distribution maps of ultrathin sections at the external surfaces of two Itokawa particles. (a) Olivine in RB-QD04-0015. (b) Low-Ca pyroxene in RB-QD04-0024. Scale bars represent $25 \mathrm{~nm}$. The nanophases forming a densely arranged array at the external surface are indicated by arrows.

been more modest than those experienced by the Itokawa particles collected at the first touchdown site that were investigated in the initial analysis.

This interpretation is consistent with the difference in the number density of solar flare tracks between the Itokawa particles investigated in this study and those in the initial analysis. Solar flare tracks were observed in RB-QD04-115, and its number density was estimated to be $<1 \times 10^{9} \mathrm{~cm}^{-2}$ (Figure 10a). On the other hand, some Itokawa particles investigated in the initial analysis show much higher track density: $1 \times 10^{10} \mathrm{~cm}^{-2}$ in RA-QD020033 and $2 \times 10^{9} \mathrm{~cm}^{-2}$ in RA-QD02-0009 (Figure 10b). By considering these results, the degrees of space weathering may be different between the two touchdown sites.

Nagao et al. (2011) also predicted the duration for solar wind to be shorter than $10^{3}$ years based on solar ${ }^{20} \mathrm{Ne}$ concentrations. Considering the solar flare track densities observed in RA-QD02-0033 corresponding to approximately $10^{4}$ years (Bradley et al. 1984), the duration for surface modification should be between $10^{3}$ and $10^{4}$ years. For the particles collected at the second touchdown site, the duration could be shorter and $<10^{3}$ years. During such a short duration, the difference in the degrees of space weathering between the two touchdown sites could have resulted.

\section{Surface modification within a single Itokawa particle}

Two TEM samples that were prepared from the opposite sides of RB-QD04-0008 do not show any detectable surface modification. On the other hand, two TEM samples that were also prepared from the opposite sides of

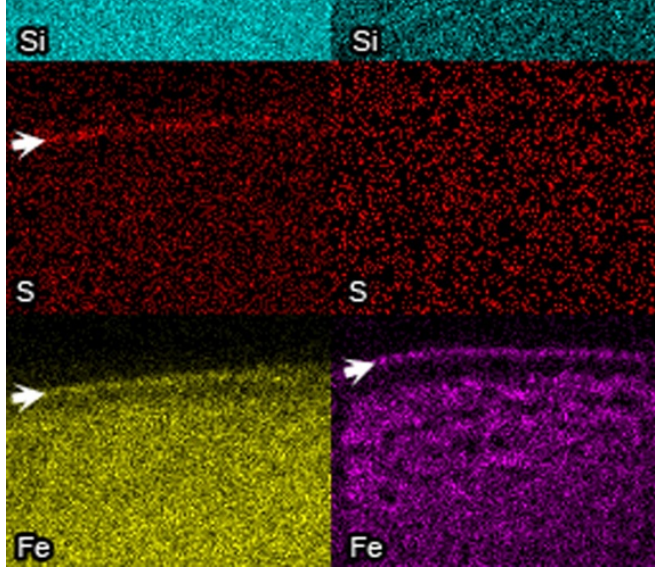




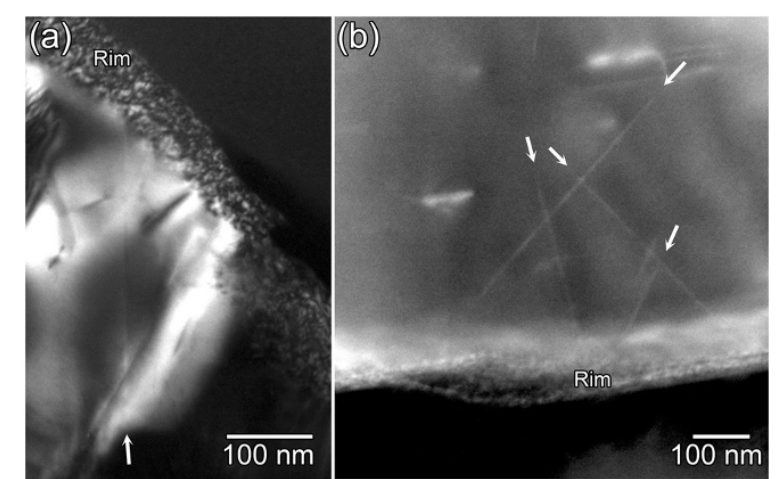

Figure 10 Dark-field images of an ultrathin section and an FIB section in two Itokawa particles. (a) Olivine in RB-QD04-0015. A solar flare track is indicated by an arrow. (b) Solar flare tracks, indicated by arrows, in low-Ca pyroxene in RA-QD02-0009, which has the vesicular composite rim (Noguchi et al. 2014). The external surfaces showing textural modification are indicated by 'Rim.'

RB-QD04-0024 have rims similar to the composite rims described in Noguchi et al. (2014), who have also reported that two TEM samples prepared from RAQD02-0032 had a composite rim. These data suggest that the Hayabusa spacecraft was able to recover Itokawa particles without significant modification and that finegrained particles on the surface of Itokawa were homogeneously irradiated by solar wind, which may be a result of the low surface gravity on Itokawa (approximately $10^{-4}$ $\mathrm{ms}^{-2}$; Hirata et al. 2009) and levitation of fine-grained particles by electrostatic repulsion caused by a photoelectron effect (Lee 1996; Hartzell and Scheeres 2013).

In the initial analysis, Nagao et al. (2011) analyzed noble gas isotopes in the Itokawa particles collected at the first touchdown. They revealed that the noble gas compositions and the release profiles vary even among the particles collected at the same touchdown site. The variety was thought to reflect different trapping sites of solar wind noble gases: Noble gases released at lower temperatures could be solar winds with lower kinetic energy and hence be trapped in the outermost layer of the grains, whereas gases released at higher temperatures could reside in deeper layers due to their higher kinetic energy. In the shallower surface layer of each grain, helium could be more enriched compared to that in the deeper surface layer, as observed in the bulk metallic glass of the Genesis (Grimberg et al. 2008). These noble gas signatures are consistent with the interpretation of our TEM results; the Itokawa particles could have been collected by the Hayabusa spacecraft as is on the Itokawa surface.

Redox state of the four Itokawa particles and comparison with that of the Tuxtuac LL5 chondrite

By measuring the Fe K-edge XANES of olivine and lowCa pyroxene in the four Itokawa samples, we checked whether they were as reduced as ordinary chondrites. We found this to be the case, and this is one more piece of evidence about the similarity to LL chondrites. The Fe $\mathrm{K}$-edge positions and the $\mathrm{Fe} \mathrm{K}$ pre-edge centroid positions of olivine, low-Ca pyroxene, and high-Ca pyroxene in the four Itokawa grains overlap with those in the Tuxtuac LL5 chondrite (Table 4). These data indicate a negligible abundance of $\mathrm{Fe}^{3+}$ ions in ferromagnesian silicates in both the Itokawa grains and equivalent Tuxtuac minerals, which is consistent with the mineralogical, petrological, and oxygen isotopic data of the Itokawa grains investigated in the initial analyses (Nakamura et al. 2011, 2014; Tsuchiyama et al. 2011, 2014; Nakamura et al. 2012; Yurimoto et al. 2011; Nakashima et al. 2013).

None of the Itokawa particles investigated in this study contain pentlandite inclusions, which is consistent with the low abundance of pentlandite in LL chondrites $(<<1 \%)$ (Graham et al. 1988; Jamsja and Ruzicka 2010).

\section{Relationships between Fo mol\% estimated by Raman spectroscopy and Fo mol\% obtained by EPMA}

Forsterite mol\% estimated by Raman spectroscopy in this study is $\mathrm{Fo}_{80}$, which is 10 percentage points higher than the Fo mol\% obtained by EPMA (Figures 3 and 4). On the other hand, Fo mol\% estimated by Raman spectroscopy in Noguchi et al. (2012) was consistent with that obtained by EPMA in Noguchi et al. (2012). The wavelength of the excitation laser is different between these studies. A green laser $(\lambda=532 \mathrm{~nm})$ was applied in this study, while a near infrared laser $(\lambda=785 \mathrm{~nm})$ was used in Noguchi et al. (2012). Some Raman shift peaks are excited from epoxy by a green laser. On the other hand, no significant peaks are excited from epoxy by a near infrared laser. Because a broad peak from epoxy (approximately $830 \mathrm{~cm}^{-1}$ ) overlaps with the olivine doublet centered at approximately $830 \mathrm{~cm}^{-1}$, it is likely that the overlap reflects the deconvolution of the doublet.

\section{Conclusions}

We investigated four Itokawa particles collected from the first touchdown site. They are indistinguishable from LL6 chondrites based on Raman spectroscopy, EPMA, and XANES analyses. The space weathered rims on them are observed but less developed on the Itokawa particles collected from the second touchdown site. This result may be consistent with the difference of the solar flare track density between the particles collected during the first touchdown and those collected during the second touchdown. However, a further study is needed to confirm this estimation because of the limited numbers of the particles investigated in this study.

\section{Competing interests}

The authors declare that they have no competing interests. 


\section{Authors' contributions}

The manuscript was written by TN and verified for accuracy by all authors. TN performed ultramicrotomy and TEM observation. JCB, LJH, and SG obtained Fe XANES data. TN, MK, TH, MK, and JPB performed STEM observation. TN, TN, RO, MU, TY, YK, MA, and TO prepared Itokawa samples. $\mathrm{RO}$ supported FE-EPMA analysis. MT performed FIB sample preparation. HK provided information of XANES of ferromagnesian silicates. All authors read and approved the final manuscript.

\section{Acknowledgements}

We thank the Hayabusa project team for the return sample. We express our thanks to N. Mori, I. Ohnishi, and K. Shimada for supporting to use FE-EPMA and FE-TEM at JEOL Corporation and Kyushu University. L. P. Keller was appreciated for the discussion on the track density at the Hayabusa symposium. We are grateful for T. Mikouchi's and A. Tsuchiyama's valuable referee comments. T. Noguchi was supported by JSPS KAKENHI grant number 2424408. J Bridges and L. Hicks were supported by STFC, UK.

\section{Author details}

${ }^{1}$ Faculty of Arts and Science, Kyushu University, 744 Motooka, Nishi-ku, Fukuoka 819-0395, Japan. ${ }^{2}$ Space Science Centre, Department of Physics and Astronomy, University of Leicester, Leicester LE1 7RH, UK. ${ }^{3}$ College of Science, Ibaraki University, 2-1-1 Bunkyo, Mito 310-8512, Japan. ${ }^{4}$ Hitachi High-Technologies Co., 882 Ichige, Hitachinaka, Ibaraki 312-8504, Japan. ${ }^{5}$ University of Hawai'i at Manoa, Hawai'i Institute of Geophysics and Planetology, 1680 East-west Road, POST 527, Honolulu, HI 96822, USA. ${ }^{6}$ Department of Earth and Planetary Sciences, Kyushu University, Hakozaki, Fukuoka 812-8581, Japan. ${ }^{7}$ Institute of Space and Astronautical Science, Japan Aerospace Exploration Agency, 3-1-1 Yoshinodai, Sagamihara, Kanagawa 252-5210, Japan. ${ }^{8}$ Division of Earth and Planetary Materials Science, Graduate School of Science, Tohoku University, 6-3 Aoba, Aramaki, Aoba-ku, Sendai 980-8578, Japan. ${ }^{9}$ Geochemical Research Center, Graduate School of Science, The University of Tokyo, 7-3-1 Hongo, Bunkyo, Tokyo 113-0033, Japan.

\section{Received: 23 May 2014 Accepted: 4 September 2014} Published: 9 October 2014

\section{References}

Binzel RP, Rivkin AS, Bus SJ, Sunshine JM, Burbine TH (2001) MUSES-C target asteroid (25143) 1998 SF36: a reddened ordinary chondrite. Meteorit Planet Sci 36:1167-1172

Bradley JP, Brownlee DE, Fraundorf P (1984) Discovery of nuclear tracks in interplanetary dust. Science 226:1432-1434

Graham AM, Christophe Michel-Levy M, Danon J, Easton AJ (1988) The Tuxtuac, Mexico, meteorite, an LL5 Chondrite fall. Meteoritics 23:321-323

Grimberg A, Baur H, Bühler F, Bochsler P, Wieler R (2008) Solar wind helium, neon, and argon isotopic and elemental composition: data from the metallic glass flown on NASA's Genesis mission. Geochim Cosmochim Acta 72:626-645

Hartzell CM, Scheeres DJ (2013) Dust levitation on asteroids and the Moon. J Geophy Res Planet 118:116-125

Hirata N, Barnouin-Jha OS, Honda C, Nakamura R, Miyamoto H, Sasaki S, Demura H, Nakamura AM, Michikami T, Gaskell RW, Saito J (2009) A survey of possible impact structures on 25143 Itokawa. Icarus 200:486-502

Hiroi T, Abe M, Kitazato K, Abe S, Clark BE, Sasaki S, Ishiguro M, Barnouin-Jhaet OS (2006) Developing space weathering on the asteroid 25143 Itokawa. Nature 443:56-58

Igarashi S, Muto S, Tanabe T (2002) Surface blistering of ion irradiated SiC studied by grazing incidence electron microscopy. J Nucl Mater 307-311:1126-1129

Ishiguro M, Hiroi T, Tholen DJT, Sasaki S, Ueda Y, Nimura T, Abe M, Clark BE, Yamamoto A, Yoshida F, Nakamura R, Hirata N, Miyamoto H, Yokota Y, Hashimoto T, Kubota T, Nakamura AM, Gaskell RW, Saito J (2007) Global mapping of the degree of space weathering on asteroid 25143 Itokawa by Hayabusa/AMICA observations. Meteorit Planet Sci 42:1791-1800

Jamsja N, Ruzicka A (2010) Shock and thermal history of Northwest Africa 4859, an annealed impact-melt breccia of $L L$ chondrite parentage containing unusual igneous features and pentlandite. Meteorit Planet Sci 45:828-849

Kuebler KE, Jolliff BL, Wang A, Haskin LA (2006) Extracting olivine (Fo-Fa) compositions from Raman spectral peak positions. Geochim Cosmochim Acta 70:6201-6222

Lee P (1996) Dust levitation on asteroids. Icarus 124:181-194

Matsumoto T, Tsuchiyama A, Gucsik A, Noguchi R, Matsuno J, Nagano T, Imai Y, Shimada A, Uesugi M, Uesugi K, Nakano T, Takeuchi A, Suzuki Y, Nakamura T,
Noguchi T, Mukai T, Abe M, Yada T, Fujimura A (2012) Micro-structure of particle surfaces of Itokawa regolith and LL chondrite fragments. 43rd Lunar Planet Sci Conf. Abstract \#1969 CD-ROM

McSween HY, Jr, Patchen AD (1989) Pyroxene thermobarometry in LL-group chondrites and implications for parent body metamorphism. Meteoritics 24:219-226

Nagao K, Okazaki R, Nakamura T, Miura YN, Osawa T, Bajo K, Matsuda S, Ebihara M, Ireland TR, Kitajima F, Naraoka H, Noguchi T, Tsuchiyama A, Yurimoto H, Zolensky ME, Uesugi M, Shirai K, Abe M, Yada T, Ishibashi Y, Fujimura A, Mukai T, Ueno M, Okada T, Yoshikawa M, Kawaguchi J (2011) Irradiation history of Itokawa regolith material deduced from noble gases in the Hayabusa samples. Science 333:1128-1131

Nakamura T, Noguchi T, Tanaka M, Zolensky ME, Kimura M, Tsuchiyama A, Nakato A, Ogami T, Ishida H, Uesugi M, Yada T, Shirai K, Fujimura A, Okazaki R, Sandford SA, Ishibashi Y, Abe M, Okada T, Ueno M, Mukai T, Yoshikawa M, Kawaguchi J (2011) Itokawa dust particles: a direct link between S-type asteroids and ordinary chondrites. Science 333:1113-1116

Nakamura E, Makishima A, Moriguti T, Kobayashi K, Tanaka R, Kunihiro T, Tsujimori T, Sakaguchi C, Kitagawa H, Ota T, Yachi Y, Yada T, Abe M, Fujimura A, Ueno M, Mukai T, Yoshikawa M, Kawaguchi J (2012) Space environment of an asteroid preserved on micrograins returned by the Hayabusa spacecraft. Proc Natl Acad Sci U S A 109:4031-4032

Nakamura T, Nakato A, Ishida H, Wakita S, Noguchi T, Zolensky ME, Tanaka M, Kimura M, Tsuchiyama A, Ogami T, Hashimoto T, Konno M, Uesugi M, Yada T, Shirai K, Fujimura A, Okazaki R, Sandford SA, Ishibashi Y, Abe M, Okada T, Ueno M, Kawaguchi J (2014) Mineral chemistry of MUSES-C Regio inferred from analysis of dust particles collected from the first- and secondtouchdown sites on asteroid Itokawa. Meteorit Planet Sci 49:215-227

Nakashima D, Kita NT, Ushikubo T, Noguchi T, Nakamura T, Valley JW (2013) Oxygen three-isotope ratios of silicate particles returned from asteroid Itokawa by the Hayabusa spacecraft: a strong link with equilibrated LL chondrites. Earth Planet Sci Lett 379:127-136

Noguchi T, Nakamura T, Kimura M, Zolensky ME, Tanaka M, Hashimoto T, Konno M, Nakato A, Ogami T, Fujimura A, Abe M, Yada T, Mukai T, Ueno M, Okada T, Shirai K, Ishibashi Y, Okazaki R (2011) Incipient space weathering observed on the surface of Itokawa dust particles. Science 333:1121-1125

Noguchi T, Kimura M, Nakamura T, Kitajima T, Tsuchiyama A, Abe M, Fujimura A, Mukai T, Okada T, Ueno M, Yada T, Ishibashi Y, Shirai K, Okazaki R (2012) An attempt to identify minerals in the Itokawa dust particles by micro-Raman spectroscopy. Bunseki Kagaku 61:299-310

Noguchi T, Kimura M, Hashimoto T, Konno M, Nakamura T, Zolensky ME, Okazak R, Tanaka M, Tsuchiyama A, Nakato A, Ogami T, Ishida H, Sagae R, Tsujimoto S, Matsumoto T, Matsuno J, Fujimura A, Abe M, Yada T, Mukai T, Ueno M, Okada T, Shirai K, Ishibashi Y (2014) Space weathered rims found on the surfaces of the Itokawa dust particles. Meteorit Planet Sci 49:188-214

Tsuchiyama A, Uesugi M, Matsushima T, Michikami T, Kadono T, Nakamura T, Uesugi K, Nakano T, Sandford SA, Noguchi R, Matsumoto T, Matsuno J, Nagano T, Imai Y, Takeuchi A, Suzuki Y, Ogami T, Katagiri J, Ebihara M, Ireland TR, Kitajima F, Nagao K, Naraoka H, Noguchi T, Okazaki R, Yurimoto H, Zolensky ME, Mukai T, Abe M, Yada T, et al. (2011) Three-dimensional structure of Hayabusa samples: origin and evolution of Itokawa regolith. Science 333:1121-1125

Tsuchiyama A, Uesugi M, Uesugi K, Nakano T, Noguchi R, Matsumoto T, Matsuno NT, Imai Y, Shimada A, Takeuchi A, Suzuki Y, Nakamura T, Noguchi T, Abe M, Yada T, Fujimura A (2014) Three-dimensional microstructure of samples recovered from asteroid 25143 Itokawa: comparison with LL5 and LL6 chondrite particles Meteorit. Planet Sci 42:172-187

Wang A, Jolliff BL, Haskin LA, Kuebler KE, Viskupic KM (2001) Characterization and comparison of structural and compositional features of planetary quadrilateral pyroxenes by Raman spectroscopy. Am Mineral 86:790-806

Williams DB, Carter CB (2009) Transmission electron microscopy: a textbook for materials science, 2nd edition. Springer, New York, NY, USA, p 760

Yurimoto H, Abe K, Abe M, Ebihara M, Fujimura A, Hashiguchi M, Hashizume K, Ireland TR, Itoh S, Katayama J, Kato C, Kawaguchi J, Kawasaki N, Kitajima F, Kobayashi S, Meike T, Mukai T, Nagao K, Nakamura T, Naraoka H, Noguchi T, Okazaki R, Park C, Sakamoto N, Seto Y, Takei M, Tsuchiyama A, Uesugi M, Wakaki S, Yada T, et al. (2011) Oxygen isotopic compositions of asteroidal materials returned from Itokawa by the Hayabusa mission. Science 333:1116-1119

\section{doi:10.1186/1880-5981-66-124}

Cite this article as: Noguchi et al:: Mineralogy of four Itokawa particles collected from the first touchdown site. Earth, Planets and Space 2014 66:124. 